Graphs and Combinatorics (2009) 25:1-14

Digital Object Identifier (DOI) 10.1007/s00373-008-0826-4

\title{
On the Existence of Elementary Abelian Cycle Systems
}

\author{
Anna Benini, Anita Pasotti \\ Dipartimento di Matematica, Facoltà di Ingegneria, Università degli Studi di Brescia, \\ Via Valotti 9, I-25133 Brescia, Italy. e-mail: anna.benini@ing.unibs.it, \\ anita.pasotti@ing.unibs.it
}

\begin{abstract}
We present some necessary and/or sufficient conditions for the existence of an elementary abelian cycle system of the complete graph. We propose a construction for some classes of perfect elementary abelian cycle systems. Finally we consider elementary abelian $k$-cycle systems of the complete multipartite graph.
\end{abstract}

Key words. (regular) ( $i$-perfect) cycle system, Partial $i$-differences.

Mathematics Subject Classification (2000) 05B30.

\section{Introduction}

Throughout this paper the standard notation of graph theory will be used, so $K_{v}$, $K_{m \times s}, C_{k}$ will denote the complete graph on $v$ vertices, the complete multipartite graph with $m$ parts of size $s$ and the $k$-cycle, respectively. Given a set $S$, by $K_{S}$ we mean the complete graph whose vertex-set is $S$.

Given two graphs $K$ and $\Gamma$, a $(K, \Gamma)$-design is a set of graphs isomorphic to $\Gamma$ whose edges partition the edge set of $K$ (see [5]). A $\left(K_{v}, C_{k}\right)$-design is also called $k$-cycle system of order $v$ (see [6]). The problem of determining the spectrum of values of $v$ for which there exists a $\left(K_{v}, C_{k}\right)$-design for a given $k$ attracted a large number of combinatorialists since the 60 's. One can easily see that necessary conditions for its existence are $v$ odd and $v(v-1)$ divisible by $2 k$. Quite recently, it has been finally proved that these conditions are also sufficient. So, there exists a k-cycle system of order $v$ if and only if $k \leq v, v$ is odd and $v(v-1) \equiv 0(\bmod 2 k)$. The if part of this theorem was solved by Alspach and Gavlas [2] in the case of $k$ odd (for another recent proof see [8]) and by S̃jna [22] and [23] in the case of $k$ even.

A $k$-cycle system of order $v$ is $i$-perfect, where $1 \leq i \leq\left\lfloor\frac{k}{2}\right\rfloor$, if for any edge $[x, y] \in K_{v}$ there is exactly one cycle of the system in which $x$ and $y$ have distance $i$. Given $I \subseteq\left\{1,2, \ldots,\left\lfloor\frac{k}{2}\right\rfloor\right\}$, by saying that a $\left(K_{v}, C_{k}\right)$-design is $I$-perfect, we mean that it is $i$-perfect for all $i \in I$. If $I=\left\{1,2, \ldots,\left\lfloor\frac{k}{2}\right\rfloor\right\}$, an $I$-perfect $\left(K_{v}, C_{k}\right)$-design is called a Steiner $k$-cycle system of order $v$ and it is denoted by $S k S(v)$. A Kirkman $k$-cycle system of order $v$, denoted by $K k S(v)$, is a $S k S(v)$ together with a partition of its cycles into 2-factors of $K_{v}$. The major known existence results about $i$-perfect 
cycle systems and, in particular, about Steiner cycle systems can be found in [1], [3], [7], [12], [13], [15], [16], [17]. Here we only recall that a necessary condition for the existence of a $S k S(v)$ is that $v$ and $k$ be odd integers.

Given an additive group $G$, a $(K, \Gamma)$-design $\mathcal{B}$ is said to be regular under $G$ (for short $G$-regular) if, up to isomorphisms, the vertices of $K$ are the elements of $G$ and whenever $B$ is a cycle of $\mathcal{B}$ we also have $B+g \in \mathcal{B}$ for every $g \in G$. Of course by $B+g$ we mean the graph defined by

$$
V(B+g)=V(B)+g \quad \text { and } \quad E(B+g)=\{[x+g, y+g] \mid[x, y] \in E(B)\} .
$$

A design is said to be cyclic, abelian, non abelian, ... when it is regular under a group having the respective property.

The existence problem of cycle systems for the cyclic case has generated a considerable amount of interest. Many authors have contributed to prove the following Theorem 1.1 which is, so far, the most important result about the existence of cyclic $k$-cycle systems (see [10], [11], [14], [18], [19], [20], [21], [24]).

Theorem 1.1. For all $v \equiv 1$ or $k(\bmod 2 k)$ there exists a cyclic $\left(K_{v}, C_{k}\right)$-design with the only definitive exceptions of $(v, k)=(9,3),(15,15),\left(p^{n}, p^{n}\right)$ with $p$ a prime and $n>1$.

In what follows we will deal with the existence problem of cycle systems for the elementary abelian case. We recall that an elementary abelian group is a finite group where every non-zero element has order a prime $p$. The only results we know about this case are the following:

Theorem 1.2 (Bonisoli, Buratti, Mazzuoccolo [7]). There exists a 2-transitive elementary abelian Kirkman $k$-cycle system of order $v$ if and only if $(k, v)=\left(p, p^{n}\right)$ for some odd prime $p$ and some positive integer $n$.

Theorem 1.3 (Granville, Moisiadis, Rees [13]). For every prime power $q=2 k e+1$ with $k$ odd, there exists an elementary abelian Steiner $k$-cycle system of order $q$.

Proof. Let $q$ be as in the statement and $g$ be a primitive element of the finite field of order $q$, then an elementary abelian Steiner $k$-cycle system of order $q$ is formed by the cycles

$$
\left(g^{i}+\alpha, g^{i+2 e}+\alpha, g^{i+2 \cdot 2 e}+\alpha, \ldots, g^{i+(k-1) \cdot 2 e}+\alpha\right)
$$

for $0 \leq i<e$ and for each field element $\alpha$.

Remark 1.4. Note that the cycles of the previous construction are nothing but the blocks of the developments ${ }^{1}$ of $C_{i}=\left(g^{i}, g^{i+2 e}, g^{i+2 \cdot 2 e}, \ldots, g^{i+(k-1) \cdot 2 e}\right)$ where $0 \leq i<e$.

1 Let $G$ be any finite additive group and $D \neq \varnothing$ any subset of $G$. The incidence structure $\operatorname{dev} D:=\{G,\{D+x, x \in G\}, \in\}$ is called the development of $D$ (see [4]). 
Also, in [25] A. Vietri proved the existence of an elementary abelian 3-cycle system of order $(2 d+1)^{2}$ when $2 d+1$ is a prime and $d \equiv 0,3,8,11(\bmod 12)$.

In Section 3 some necessary and sufficient conditions for the existence of elementary abelian $k$-cycle systems (EA- $k$-cycle systems, for short) will be proved. It is convenient to give here some notation about finite fields, since we will use them for getting our constructions.

Given a prime power $q$, the finite field of order $q$ will be denoted by $\mathbb{F}_{q}$, while $\mathbb{F}_{q}^{*}$ will denote the multiplicative group of $\mathbb{F}_{q}$. Also, we denote by $C^{e}$ the group of non-zero $e$-th powers in $\mathbb{F}_{q}, e$ being any divisor of $q-1$. The cosets of $C^{e}$ in $\mathbb{F}_{q}^{*}$, also called cyclotomic classes of index $e$, are $C^{e}=g^{0} C^{e}, g^{1} C^{e}, g^{2} C^{e}, \ldots, g^{e-1} C^{e}$, where $g$ is a primitive element of $\mathbb{F}_{q}$. For simplicity, once $g$ has been fixed, we set $C_{i}^{e}:=g^{i} C^{e}$. When $e=2$, we will write $\mathbb{F}_{q}^{\square}$ and $\mathbb{F}_{q}^{\square}$ instead of $C^{2}$ and $C_{1}^{2}$, respectively.

\section{The Method of Partial $i$-differences to Construct Regular $i$-perfect $\left(K_{v}, C_{k}\right)$-designs}

From [12] we learn that any regular $i$-perfect $k$-cycle system can be characterized by the method of partial differences introduced by M. Buratti in [9]. Here we recall some definitions and the main result from [12], useful in the following.

Definition 2.1. Let $A=\left(a_{0}, a_{1}, \ldots, a_{k-1}\right)$ be a $k$-cycle with vertices in an abelian group $G$ and let $d$ be the order of the stabilizer of $A$ under the natural action of $G$, that is $d=|\{g \in G: A+g=A\}|$. Given $i \in\{1,2, \ldots,\lfloor k / 2\rfloor\}$, the multisets

$$
\begin{aligned}
\Delta_{i} A & =\left\{ \pm\left(a_{h+i}-a_{h}\right) \mid 0 \leq h<k\right\} \\
\partial_{i} A & =\left\{ \pm\left(a_{h+i}-a_{h}\right) \mid 0 \leq h<k / d\right\}
\end{aligned}
$$

where the subscripts are taken modulo $k$, are called the list of $i$-differences from $A$ and the list of partial $i$-differences from $A$, respectively.

Of course, $d=1$ implies $\partial_{i} A=\Delta_{i} A$. More generally, given a set $\mathcal{F}$ of $k$-cycles with vertices in $G$, by $\Delta_{i} \mathcal{F}$ and $\partial_{i} \mathcal{F}$ one means the union (counting multiplicities) of all multisets $\Delta_{i} A$ and $\partial_{i} A$ respectively, where $A \in \mathcal{F}$.

Theorem 2.2. Given $I \subseteq\left\{1,2, \ldots, \frac{k}{2}\right\}$, a G-regular I-perfect $\left(K_{v}, C_{k}\right)$-design is equivalent to a set $\mathcal{F}$ of $k$-cycles with vertices in $G$ (called base cycles) such that $\partial_{i} \mathcal{F}=$ $G-\{0\}$ for all $i \in I$.

As an immediate consequence we have:

Corollary 2.3. $A$ G-regular $\left(K_{v}, C_{k}\right)$-design is equivalent to a set $\mathcal{F}$ of $k$-cycles with vertices in $G$ such that $\partial_{1} \mathcal{F}=G-\{0\}$.

In Example 2.5 we will show how to construct an EA- $\left(K_{25}, C_{15}\right)$-design just applying Corollary 2.3. For our purposes the following notation will be useful. 
Let $a_{0}, a_{1}, \ldots, a_{r-1}, x$ be elements of an additive group $G$, with $x$ of order $p$. The closed trail represented by the concatenation of the sequences

$$
\begin{gathered}
\left(a_{0}, a_{1}, \ldots, a_{r-1}\right) \\
\left(a_{0}+x, a_{1}+x, \ldots, a_{r-1}+x\right) \\
\left(a_{0}+2 x, a_{1}+2 x, \ldots, a_{r-1}+2 x\right) \\
\ldots \\
\left(a_{0}+(p-1) x, a_{1}+(p-1) x, \ldots, a_{r-1}+(p-1) x\right)
\end{gathered}
$$

will be denoted by

$$
\left[a_{0}, a_{1}, \ldots, a_{r-1}\right]_{x} .
$$

Remark 2.4. Note that $\left[a_{0}, a_{1}, \ldots, a_{r-1}\right]_{x}$ is a (pr)-cycle if and only if the elements $a_{i}$, for $i=0, \ldots, r-1$, belong to pairwise distinct cosets of the subgroup $\langle x\rangle$ in $G$. Also, if $A=\left[a_{0}, a_{1}, \ldots, a_{r-1}\right]_{x}$ is a ( $\left.p r\right)$-cycle then

$$
\partial_{1} A=\left\{ \pm\left(a_{i}-a_{i-1}\right) \mid i=1, \ldots, r-1\right\} \cup\left\{ \pm\left(a_{0}+x-a_{r-1}\right)\right\} .
$$

Example 2.5. Let $G=\mathbb{Z}_{5} \times \mathbb{Z}_{5}$ and consider the following 15-cycles with vertices in $G$ (with some abuse, let us write any pair $(x, y)$ as $x y$ ):

$$
\begin{aligned}
A & =[00,01,13]_{10}=(00,01,13,10,11,23,20,21,33,30,31,43,40,41,03) \\
B & =[00,13,22]_{01}=(00,13,22,01,14,23,02,10,24,03,11,20,04,12,21) \\
C & =[00,22,32]_{02}=(00,22,32,02,24,34,04,21,31,01,23,33,03,20,30) \\
D & =[00,11,34]_{03}=(00,11,34,03,14,32,01,12,30,04,10,33,02,13,31) .
\end{aligned}
$$

One can easily check that:

$$
\begin{array}{ll}
\partial_{1} A=\{ \pm 01, \pm 12\} \cup\{ \pm 02\} & \partial_{1} B=\{ \pm 13, \pm 14\} \cup\{ \pm 21\} \\
\partial_{1} C=\{ \pm 22, \pm 10\} \cup\{ \pm 20\} & \partial_{1} D=\{ \pm 11, \pm 23\} \cup\{ \pm 24\}
\end{array}
$$

Setting $\mathcal{F}=\{A, B, C, D\}$ we have $\partial_{1} \mathcal{F}=G-\{00\}$. Hence, by Corollary 2.3, $\{A, B, C, D\}$ is a set of base cycles of an EA-15-cycle system of order 25 .

Explicitly, the required design is the following:

$$
\{A+0 i \mid 0 \leq i \leq 4\} \cup\{B+i 0 \mid 0 \leq i \leq 4\} \cup\{C+i 0 \mid 0 \leq i \leq 4\} \cup\{D+i 0 \mid 0 \leq i \leq 4\} .
$$

\section{Existence of Elementary Abelian Cycle Systems}

We have already observed that necessary and sufficient conditions for the existence of a $k$-cycle system of order $v$ are $k \leq v, v$ odd and $v(v-1) \equiv 0(\bmod 2 k)$.

For the existence of an elementary abelian $k$-cycle system of order $v$ a stricter condition is necessary.

Proposition 3.1. If an EA-( $\left.K_{v}, C_{k}\right)$-design exists, then

a) $v=p^{n}$ where $p$ is an odd prime and $n \geq 1$;

b) $k \leq v$;

c) $p\left(p^{n}-1\right) \equiv 0(\bmod 2 k)$. 
Proof. Let $\mathcal{B}$ be the required system and let $A$ be a $k$-cycle of $\mathcal{B}$. Conditions $a$ ) and $b$ ) follow obviously. To prove condition $c$ ) one can easily see that the order of the stabilizer of $A$ under the natural action of $\mathbb{Z}_{p}^{n}$ is 1 or $p$ and this implies that the order of the orbit of $A$ is $p^{n}$ or $p^{n-1}$, respectively. Since the orbits partition the set of blocks of $\mathcal{B}$ we have that $p^{n-1}$ must be a divisor of $\frac{p^{n}\left(p^{n}-1\right)}{2 k}$, that is $\frac{p\left(p^{n}-1\right)}{2 k} \in \mathbb{Z}$.

The previous proposition suggests searching sufficient conditions distinguishing two different cases: the first one when $p$ is a divisor of $k$ and the second one in which $p$ does not divide $k$.

\subsection{Case: $p$ Divides $k$}

Lemma 3.2. Let $p$ be a prime such that $p^{n} \equiv 1(\bmod 2 t)$. If there exists a sequence $A=\left(a_{0}=0, a_{1}, a_{2}, \ldots, a_{t}\right)$ of elements of $\mathbb{F}_{p^{n}}$ such that

a) $D:=\left\{a_{i}-a_{i-1} \mid 1 \leq i \leq t\right\}$ is a complete system of representatives for the cosets of $C^{t}$ in $\mathbb{F}_{p^{*}}^{*}$

b) $\left\{a_{i}-a_{j} \mid 0 \leq i<j \leq t-1\right\} \cap\left\{h a_{t} \mid 0 \leq h \leq p-1\right\}=\emptyset$

then there exists an EA-(pt)-cycle system of order $p^{n}$.

Proof. Let $B=\left[0, a_{1}, a_{2}, \ldots, a_{t-1}\right]_{a_{t}}$. Condition $\left.b\right)$ together with Remark 2.4 make us sure that $B$ is a $(p t)$-cycle. Again by Remark 2.4, we obtain $\partial_{1} B=\langle-1\rangle \cdot D$. Now, let $S$ be a complete system of representatives for the cosets of $\langle-1\rangle$ in $C^{t}$ and let $\mathcal{F}=\{s \cdot B \mid s \in S\}$. Since $\partial_{1} \mathcal{F}=S \cdot \partial_{1} B=S \cdot\langle-1\rangle \cdot D=C^{t} \cdot D$, by Condition $\left.a\right)$ it follows that $\partial_{1} \mathcal{F}=\mathbb{F}_{p^{n}}^{*}$, hence, by Corollary 2.3, we have that $\mathcal{F}$ is the set of base cycles of an EA- $(p t)$-cycle system of order $p^{n}$.

Proposition 3.3. Let $p$ be a prime. There exists an EA-(pt)-cycle system of order $p^{n}$ for all $p^{n} \equiv 1(\bmod 2 t)$ such that

$$
\frac{p^{n}-1}{p-1} \geq \frac{t^{2}(t-1)}{2} .
$$

Proof. We consider the sequence $A=\left(a_{0}=0, a_{1}, \ldots, a_{t}\right)$ of elements of $\mathbb{F}_{p^{n}}$ so defined: for all $i=1, \ldots, t-1$ we set

$$
\begin{aligned}
& a_{i}=a_{i-1}+x_{i-1} \text { with } x_{i-1} \in B_{i-1}:=C_{i-1}^{t}-A_{i-1} \\
& \text { where } A_{i-1}=\left\{a_{j}-a_{k} \mid 0 \leq j<k \leq i-1\right\},
\end{aligned}
$$

and

$$
\begin{aligned}
& a_{t}=a_{t-1}+x_{t-1} \text { with } x_{t-1} \in B_{t-1}:=C_{t-1}^{t}-A_{h} \\
& \text { where } A_{h}=\left\{h\left(a_{i}-a_{j}\right)-a_{t-1} \mid 0 \leq h \leq p-1,0 \leq i<j \leq t-1\right\} .
\end{aligned}
$$

Firstly, we prove that such a sequence exists, namely that $B_{i-1} \neq \emptyset$ for all $i=$ $1, \ldots, t$. By hypothesis $\frac{p^{n}-1}{t} \geq(p-1) \frac{t(t-1)}{2}$, which implies $\left|C^{t}\right| \geq(p-1) \frac{t(t-1)}{2}$. It 
is easy to check that $\left|A_{i-1}\right|=\frac{i(i-1)}{2} \leq \frac{(t-1)(t-2)}{2}$, so $B_{i-1} \neq \emptyset$ for all $i=1, \ldots, t-1$. Moreover, $\left|A_{h}\right| \leq(p-1) \frac{t(t-1)}{2}+1$ and note that, for instance, 0 and $-x_{0}$ are two elements of $A_{h}$ which do not lie in $C_{t-1}^{t}$. Hence $B_{t-1} \neq \varnothing$ too.

Now we have to prove that the sequence $A$ defined as above satisfies Conditions a) and $b$ ) of Lemma 3.2.

Since $D:=\left\{a_{i}-a_{i-1} \mid 1 \leq i \leq t\right\}=\left\{x_{0}, x_{1}, \ldots, x_{t-1}\right\}$, where $x_{i} \in C_{i}^{t}$ for any $i=0, \ldots, t-1$, Condition $a)$ is satisfied.

Now we deal with Condition $b$ ). Reasoning by contradiction, we suppose that there exist $h, i, j$ with $0 \leq h \leq p-1,0 \leq i<j \leq t-1$ such that $a_{i}-a_{j}=$ $h a_{t}=h\left(a_{t-1}+x_{t-1}\right)$, that is $h x_{t-1}=\left(a_{i}-a_{j}\right)-h a_{t-1}$. If $h \neq 0$ then $x_{t-1}=$ $h^{-1}\left(a_{i}-a_{j}\right)-a_{t-1}$, but $x_{t-1} \in B_{t-1}$. A contradiction. If $h=0$ then $a_{i}=a_{j}$ implies $x_{j-1}=a_{i}-a_{j-1}$, again a contradiction, since $x_{j-1} \in B_{j-1}$.

We observe that the previous Condition (1) is always satisfied when $t<n$. In this case, obviously under the necessary condition $p^{n} \equiv 1(\bmod 2 t)$, we are able to give a concrete construction for an EA-cycle system.

Construction 3.4. Case $\mathbf{t}<\mathbf{n}$. We consider the sequence $A=\left(a_{0}=0, a_{1}, a_{2}, \ldots, a_{t}\right)$ defined by the rule $a_{i}=a_{i-1}+g^{i-1}$ for $i=1, \ldots, t$, where $g$ is a primitive element of $\mathbb{F} p^{n}$. We want to prove that $B=\left[a_{0}, a_{1}, a_{2}, \ldots, a_{t-1}\right]_{a_{t}}$ is a $(p t)$-cycle. By Remark 2.4, it is sufficient to show that the elements of $\left\{a_{0}, a_{1}, \ldots, a_{t-1}\right\}$ belong to different cosets of the additive group $\left\langle a_{t}\right\rangle$. Reasoning by contradiction, let $i, j$ with $0 \leq i<j \leq t-1$ such that $a_{i}-a_{j}=\alpha a_{t}$ for a suitable $\alpha \in \mathbb{F}_{p}$. Namely $g^{j}+\ldots+g^{i-1}=\alpha\left(1+g+\ldots+g^{t-1}\right)$, but it cannot happen since $g$ is primitive and $t<n$. It is easy to see that $\partial_{1} B=\langle-1\rangle \cdot\left\{1, g, g^{2}, \ldots, g^{t-1}\right\}$. Hence, by taking a complete system $S$ of representatives for the cosets of $\langle-1\rangle$ in $C^{t}$, using Corollary 2.3 it follows that $\mathcal{F}=\{s \cdot B \mid s \in S\}$ is the set of base cycles for an EA- $\left(K_{p^{n}}, C_{p t}\right)$-design.

Proposition 3.5. Let $q=p^{d r}$ be an odd prime power, where $r>1$. There exists an $E A$ - $(p t)$-cycle system of order $q$ for every odd integer $t$ dividing $p^{d}-1$.

Proof. Let $q-1=p^{n}-1=2 e t$, with $t$ as in the statement. Let $g$ be a primitive element of $\mathbb{F}_{q}$ and set $\varepsilon=g^{2 e}$. Starting from the construction of Theorem 1.3, now we are able to construct an EA- $(p t)$-cycle system of order $q$.

We consider the closed trail $C_{i}=\left[g^{i}, g^{i} \varepsilon, g^{i} \varepsilon^{2}, \ldots, g^{i} \varepsilon^{t-1}\right]_{x_{i}}$, where $x_{i}=g^{i}(g-1)$ $\left(1-\varepsilon^{t-1}\right)$ and $i$ is taken modulo $e$. We want to prove that $C_{i}$ is a $(p t)$-cycle for all $i \in \mathbb{Z}_{e}$. By Remark 2.4, it is sufficient to show that the elements of $\left\{g^{i}, g^{i} \varepsilon, g^{i} \varepsilon^{2}, \ldots\right.$, $\left.g^{i} \varepsilon^{t-1}\right\}$ belong to different cosets of the additive group $<x_{i}>$ for all $i \in \mathbb{Z}_{e}$. In other words, we have to prove that, for any $\lambda \in \mathbb{F}_{p}$ and for any $0 \leq j<k \leq t-1$

$$
g^{i}\left(\varepsilon^{k}-\varepsilon^{j}\right) \neq \lambda g^{i}(g-1)\left(1-\varepsilon^{t-1}\right) \quad \forall i=0, \ldots, e-2
$$

and also

$$
g^{e-1}\left(\varepsilon^{k}-\varepsilon^{j}\right) \neq \lambda\left(1-g^{e-1}\right)\left(1-\varepsilon^{t-1}\right) .
$$


Reasoning by contradiction, we assume that in (2) the equality holds for some $\lambda \in \mathbb{F}_{p}$ and some $0 \leq j<k \leq t-1$. If $\lambda=0$, then $\varepsilon^{k}=\varepsilon^{j}$ results in a contradiction. If $\lambda \neq 0$, we obtain $g=\lambda^{-1} \frac{\varepsilon^{k}-\varepsilon^{j}}{1-\varepsilon^{t-1}}+1$, hence $g \in \mathbb{F}_{p}(\varepsilon)$. Since $g$ is a primitive element of $\mathbb{F}_{q}$ we obtain $\mathbb{F}_{p}(\varepsilon)=\mathbb{F}_{q}$. On the other hand, by hypothesis, $\frac{p^{n}-1}{p^{d}-1}$ divides $2 e$ and $\frac{p^{n}-1}{p^{d}-1} \neq 1$. So $g^{2 e}=\varepsilon$ belongs to the proper subfield of $\mathbb{F}_{q}$ of order $p^{d}$, that is $\mathbb{F}_{p}(\varepsilon) \subset \mathbb{F}_{q}$, again a contradiction.

In a similar way we can show that (3) is also satisfied.

Now, we consider the set $\mathcal{F}=\left\{C_{i} \mid i \in \mathbb{Z}_{e}\right\}$. It is easy to check that $\partial_{1} \mathcal{F}=\mathbb{F}_{q}^{*}$, thus, by Corollary $2.3, \mathcal{F}$ is the set of base cycles of an EA- $(p t)$-cycle system of order $q$.

There exist parameters which satisfy Proposition 3.3 but not Proposition 3.5 (see Table 1) and also vice versa (see Table 2).

On the other hand, Proposition 3.3 and Proposition 3.5 are not complementary. In fact there are EA-cycle systems whose existence is guaranteed by both these results (see Table 3 ) and others whose existence is guaranteed by neither Proposition 3.3 nor Proposition 3.5 (see Table 4).

Of course Condition (1) of Proposition 3.3 is certainly satisfied when $t=$ 1 (see also Theorem 1.2) and $t=2$. Moreover, if $t=3$ Proposition 3.3 gives the existence of an EA-3p-cycle system of order $p^{n}$ for every prime $p$ such that $p^{n} \equiv 1(\bmod 6)$ with $(p, n) \neq(5,2),(7,2)$. On the other hand, we have a direct construction for an EA-15-cycle systems of order 25 (see Example 2.5) and

Table 1.

\begin{tabular}{rrrrrrrrrrrrrrr}
\hline$p$ & 3 & 5 & 7 & 11 & 11 & 13 & 13 & 13 & 17 & 17 & 17 & 23 & 23 & 23 \\
$n$ & 6 & 5 & 4 & 6 & 6 & 4 & 8 & 8 & 4 & 8 & 8 & 4 & 6 & 6 \\
$t$ & 7 & 11 & 5 & 45 & 63 & 15 & 238 & 510 & 15 & 290 & 870 & 5 & 91 & 143 \\
\hline
\end{tabular}

Table 2.

\begin{tabular}{lrrrrrrrrrrrrr}
\hline$p$ & 3 & 5 & 7 & 7 & 7 & 7 & 7 & 11 & 11 & 19 & 19 & 19 & 19 \\
$n$ & 6 & 6 & 2 & 6 & 6 & 10 & 10 & 2 & 4 & 2 & 4 & 6 & 6 \\
$t$ & 13 & 31 & 3 & 57 & 171 & 2801 & 8403 & 5 & 15 & 9 & 45 & 381 & 3429 \\
$d$ & 3 & 3 & 1 & 3 & 3 & 5 & 5 & 1 & 2 & 1 & 2 & 3 & 3 \\
\hline
\end{tabular}

Table 3.

\begin{tabular}{llllllrrrrrrrr}
\hline$p$ & 3 & 5 & 7 & 7 & 7 & 7 & 11 & 11 & 11 & 19 & 19 & 19 & 19 \\
$n$ & 8 & 4 & 3 & 6 & 6 & 6 & 6 & 6 & 6 & 4 & 4 & 4 & 4 \\
$t$ & 5 & 3 & 3 & 3 & 9 & 19 & 3 & 5 & 15 & 3 & 5 & 9 & 15 \\
$d$ & 4 & 2 & 1 & 3 & 3 & 3 & 2 & 2 & 2 & 2 & 2 & 2 & 2 \\
\hline
\end{tabular}

Table 4.

\begin{tabular}{rrrrrrrrrrrr}
\hline$p$ & 3 & 3 & 5 & 5 & 5 & 7 & 7 & 7 & 11 & 11 & 13 \\
$n$ & 4 & 8 & 2 & 4 & 8 & 4 & 6 & 8 & 6 & 6 & 4 \\
$t$ & 5 & 41 & 3 & 13 & 313 & 15 & 43 & 1201 & 105 & 315 & 119 \\
\hline
\end{tabular}


Proposition 3.5 ensures the existence of an EA-21-cycle system of order 49. Besides, if we consider the case of $t=4$, again Proposition 3.3 ensures the existence of an EA-4 $p$-cycle system of order $p^{n}$ for every prime $p$ such that $p^{n} \equiv 1(\bmod 8)$ with $(p, n) \neq(5,2),(7,2),(11,2),(13,2),(17,2),(19,2)$. Anyway, we have directly checked the existence of such cycle systems. Hence we can conclude with the following:

Proposition 3.6. There exists an EA-(pt)-cycle system of order $p^{n}$ for every prime $p$ such that $p^{n} \equiv 1(\bmod 2 t)$ with $t=1,2,3,4$ and $t \leq p^{n-1}$.

\subsection{Case: $p$ Does Not Divide $k$}

In the following, by adapting a known construction for perfect cyclic cycle systems (see [12]), we are able to give a construction method for perfect elementary abelian $k$-cycle systems of order $p^{n}$ where $p$ is not a divisor of $k$. Note that in this case condition $c)$ of Proposition 3.1 becomes $p^{n} \equiv 1(\bmod 2 k)$. Hence we consider only the case of $k$ even, since the case of $k$ odd has already been solved (see Theorem 1.3).

Construction 3.7. Let $q=p^{n} \equiv 1(\bmod 2 k)$ be a prime power, where $k=2^{e} t$ with $e \geq 1$ and $t$ odd. Let $\varepsilon$ be a primitive $t$-th root of unity in $\mathbb{F}_{q}$ and let $\left(x_{0}, x_{1}, \ldots, x_{2^{e}-1}\right)$ be a $2^{e}$-tuple of elements of $\mathbb{F}_{q}^{*}$ belonging to pairwise distinct cosets of $\langle\varepsilon\rangle$. Let $A=\left(a_{0}, a_{1}, \ldots, a_{k-1}\right)$ be the $k$-cycle defined by the rule

$$
a_{j}=\varepsilon^{s} x_{r} \quad \text { for } j=2^{e} s+r, \quad 0 \leq r<2^{e}, \quad 0 \leq j \leq k-1
$$

or, more explicitly,

$$
A=\left(x_{0}, x_{1}, \ldots, x_{2^{e}-1}, \varepsilon x_{0}, \varepsilon x_{1}, \ldots, \varepsilon x_{2^{e}-1}, \ldots, \varepsilon^{t-1} x_{0}, \varepsilon^{t-1} x_{1}, \ldots, \varepsilon^{t-1} x_{2^{e}-1}\right) .
$$

Now, for any $i=1, \ldots, k / 2$, we consider the multiset $\Delta_{i} A$ of all the $i$-differences of $A$ and the list $L_{i}$ of the first $2^{e} i$-differences, that is $L_{i}=\left\{a_{h+i}-a_{h} \mid 0 \leq h \leq 2^{e}-1\right\}$. It is easy to see that $\Delta_{i} A=\langle-\varepsilon\rangle \cdot L_{i}$, where $\langle-\varepsilon\rangle$ is the group of $2 t$-th roots of unity in $\mathbb{F}_{q}$.

Let $I$ be a subset of $\left\{1,2, \ldots, \frac{k}{2}\right\}$ containing 1 . If $L_{i}$ results in a complete system of representatives for the cosets of $C^{2^{e}}$ in $\mathbb{F}_{q}^{*}$ for all $i \in I$ then, taking a complete system $S$ of representatives for the cosets of $\langle-\varepsilon\rangle$ in $C^{2^{e}}$ and setting $\mathcal{F}=\{s \cdot A \mid s \in S\}$, we have $\Delta_{i} \mathcal{F}=S \cdot \Delta_{i} A=S \cdot\langle-\varepsilon\rangle \cdot L_{i}=C^{2^{e}} \cdot L_{i}=\mathbb{F}_{q}^{*}$. Hence, by Theorem 2.2 (note that in this case $\partial_{i} A=\Delta_{i} A$ ), $\mathcal{F}$ is the set of base cycles of an $I$-perfect $\operatorname{EA-}\left(K_{q}, C_{k}\right)$-design.

Of course, applying Construction 3.7 is the more difficult the larger $e$ and $|I|$ are. We are able to obtain some results in the following two different cases: $(e=$ $\left.1, I=\left\{1,2,4,6, \ldots, \frac{k}{2}-1\right\}\right)$ and $(e>1, I=\{1\})$. 


\subsubsection{Case $e=1$}

Proposition 3.8. Let $q=p^{n} \equiv 1(\bmod 2 k)$ be a prime power and let $I=\{1,2,4$, $\left.6, \ldots, \frac{k}{2}-1\right\}$. For all $k \equiv 2(\bmod 4)$, with $k \neq 2$, there exists an I-perfect $E A$ $\left(K_{q}, C_{k}\right)$-design.

Proof. Let $k=2 t, t$ odd, and let $\varepsilon$ be a primitive $k / 2$-th root of unity in $\mathbb{F}_{q}$. Following Construction 3.7, we have to find a pair of elements from two different cosets of $\langle\varepsilon\rangle \subseteq \mathbb{F}_{q}^{*}$, say $\left(x_{0}, x_{1}\right)$, in such a way that $x_{1}-x_{0}$ and $\varepsilon x_{0}-x_{1}$ belong to different cosets of $C^{2}=\mathbb{F}_{q}^{\square} \subseteq \mathbb{F}_{q}^{*}$. We want to prove that there exists an $a \in \mathbb{F}_{q}^{\square}$ such that $\left(1, \frac{a+\varepsilon}{a+1}\right)$ is a suitable pair.

Firstly, we observe that $x_{0}=1$ belongs to $\langle\varepsilon\rangle$ and we can always find an $x_{1}=$ $\frac{a+\varepsilon}{a+1} \notin\langle\varepsilon\rangle$, because $\left|\left\{\frac{a+\varepsilon}{a+1} \mid a \in \mathbb{F}_{q}^{\nabla}\right\}\right|=\frac{q-1}{2}>\frac{k}{2}=|\langle\varepsilon\rangle|$. Secondly, we note that $x_{1}-x_{0}=\frac{\varepsilon-1}{a+1}$ and $\varepsilon x_{0}-x_{1}=a \frac{\varepsilon-1}{a+1}$ belong to different cosets of $\mathbb{F}_{q}^{\square}, a$ being in $\mathbb{F}_{q}^{\square}$. At this point, Construction 3.7 makes us sure that

$$
\mathcal{F}=\left\{s\left(1, x_{1}, \varepsilon, \varepsilon x_{1}, \varepsilon^{2}, \varepsilon^{2} x_{1}, \ldots, \varepsilon^{k / 2-1}, \varepsilon^{k / 2-1} x_{1}\right) \mid s \in S\right\}
$$

is the set of base cycles of an EA- $\left(K_{q}, C_{k}\right)$-design, $S$ being a complete system of representatives for the cosets of $\langle-\varepsilon\rangle$ in $\mathbb{F}_{q}^{\square}$.

Finally, note that if $x_{1} \in \mathbb{F}_{q}^{\square}$ then the design is $I$-perfect, where $I=\{1,2,4,6, \ldots$, $k / 2-1\}$. In fact for any $i \in I$ we have

$$
\Delta_{i} \mathcal{F}=S \cdot<-\varepsilon>\cdot\left\{x_{1}\left(\varepsilon^{i / 2}-1\right), \varepsilon^{i / 2}-1\right\}=\mathbb{F}_{q}^{\square} \cdot\left\{x_{1}\left(\varepsilon^{i / 2}-1\right), \varepsilon^{i / 2}-1\right\}=\mathbb{F}_{q}^{*} .
$$

Actually, we can prove that such a choice is always possible. In fact, we know that $x_{1} \in A=\left\{\frac{a+\varepsilon}{a+1} \mid a \in \mathbb{F}_{q}^{\nabla}\right\}$ and that $A$ has size $\frac{q-1}{2}$. By way of contradiction we assume that all the elements of $A$ are squares, so $A=\mathbb{F}_{q}^{\square}$ since they have the same size. But 1 is a square which does not lie in $A$. The assertion follows.

\section{Example 3.9. $\{1,2,4\}$-perfect EA- $\left(K_{81}, C_{10}\right)$-design}

As an explicit example, we apply the above proposition to get a $\{1,2,4\}$-perfect EA- $\left(K_{81}, C_{10}\right)$-design.

In $\mathbb{F}_{81}=\mathbb{Z}_{3}[x] /\left\langle x^{4}+x+2\right\rangle$ we choose $x$ as a primitive element and $\varepsilon=x^{16}=2 x^{3}+$ $x+2$ as a primitive 5 -th root of unity in $\mathbb{F}_{81}$. As in Proposition 3.8 we set: $x_{0}=1 \in\langle\varepsilon\rangle$, $a=2 x^{3}+x^{2}+x=x^{75} \in \mathbb{F}_{81}^{7}$ and, consequently, $x_{1}=\frac{a+\varepsilon}{a+1}=x \notin\langle\varepsilon\rangle$. Moreover, we note that $x_{1}-x_{0}=x+2=x^{44} \in \mathbb{F}_{81}^{\square}$ while $\varepsilon x_{0}-x_{1}=2 x^{3}+2=x^{39} \in \mathbb{F}_{81}^{\square}$. So, considering the cycle

$$
C=\left(1, x, \varepsilon, \varepsilon x, \varepsilon^{2}, \varepsilon^{2} x, \varepsilon^{3}, \varepsilon^{3} x, \varepsilon^{4}, \varepsilon^{4} x\right)
$$

and assuming the set $S=\left\{1, x^{2}, x^{4}, x^{6}\right\}$ as a complete system of representatives for the cosets of $\langle-\varepsilon\rangle$ in $\mathbb{F}_{81}^{\square}$, we can say that $\mathcal{F}=\left\{C, x^{2} C, x^{4} C, x^{6} C\right\}$ is a set of base cycles of an EA- $\left(K_{81}, C_{10}\right)$-design $\mathcal{B}$. Also, since $x_{1}=x$ is a non-square, $\mathcal{B}$ is a $\{1,2,4\}$-perfect EA-10-cycle system of order 81 . 


\subsubsection{Case $e>1$}

Proposition 3.10. Let $q \equiv 1(\bmod 2 k)$ be a prime power, where $k=2^{e} t$ with $e>1$ and $t$ odd. There exists an EA-k-cycle system of order $q$ for any $q>2^{2 e-1}\left[\left(2^{e}-1\right) t-\right.$ $\left.\left(2^{e}-3\right)\right]-2^{e+1}+1$.

Proof. Let $g$ be a primitive element of $\mathbb{F}_{q}$ and let $\varepsilon$ be a primitive $t$-th root of unity in $\mathbb{F}_{q}$. Let $\left(\bar{x}_{1}, \bar{x}_{2}, \ldots, \bar{x}_{2^{e}-1}\right)_{c}$ be the solution of the system

$$
\left\{\begin{array}{l}
x_{2}-x_{1}=g\left(x_{1}-1\right) c \\
x_{3}-x_{2}=g^{2}\left(x_{1}-1\right) c \\
\vdots \\
x_{2^{e}-1}-x_{2^{e}-2}=g^{2^{e}-2}\left(x_{1}-1\right) c \\
\varepsilon-x_{2^{e}-1}=g^{2^{e}-1}\left(x_{1}-1\right) c
\end{array}\right.
$$

where $c$ is a fixed element of $C^{2^{e}}$. Note that such a solution exists if and only if $D=1+c \sum_{h=1}^{2^{e}-1} g^{h} \neq 0$. In this case, for any $i=1, \ldots, 2^{e}-1$ we have

$$
\bar{x}_{i}=\frac{\varepsilon+\varepsilon c \sum_{h=1}^{i-1} g^{h}+c \sum_{h=i}^{2^{e}-1} g^{h}}{D} .
$$

It is not hard to see that $\left(\bar{x}_{1}, \bar{x}_{2}, \ldots, \bar{x}_{2^{e}-1}\right)_{c_{1}}=\left(\bar{x}_{1}, \bar{x}_{2}, \ldots, \bar{x}_{2^{e}-1}\right)_{c_{2}}$ if and only if $c_{1}=c_{2}$. So, for any fixed $c$ in $C^{2^{e}}$ provided $D \neq 0$, the set $L_{1}=\left\{\bar{x}_{1}-1, \bar{x}_{2}-\right.$ $\left.\bar{x}_{1}, \ldots, \bar{x}_{2^{e}-1}-\bar{x}_{2^{e}-2}, \varepsilon-\bar{x}_{2^{e}-1}\right\}$ results in a complete system of representatives for the cosets of $C^{2^{e}}$ in $\mathbb{F}_{q}^{*}$. Now, let $A$ be the closed trail

$$
A=\left(1, \bar{x}_{1}, \ldots, \bar{x}_{2^{e}-1}, \varepsilon, \varepsilon \bar{x}_{1}, \ldots, \varepsilon \bar{x}_{2^{e}-1}, \ldots, \varepsilon^{t-1}, \varepsilon^{t-1} \bar{x}_{1}, \ldots, \varepsilon^{t-1} \bar{x}_{2^{e}-1}\right) .
$$

We want to prove that $A$ is a $k$-cycle with vertices in $\mathbb{F}_{q}^{*}$, namely that there exists an element $\bar{c} \in C^{2^{e}}$ such that $D \neq 0, \bar{x}_{\ell} \neq 0$ for any $\ell=1, \ldots, 2^{e}-1$, and $\bar{x}_{\ell}, \bar{x}_{i} \cdot \bar{x}_{j}^{-1} \notin\langle\varepsilon\rangle$ for any $\ell=1, \ldots, 2^{e}-1$ and $1 \leq j<i \leq 2^{e}-1$. Note that, for any fixed $\ell=1, \ldots, 2^{e}-1$, there is exactly one value of $c \in C^{2^{e}}$ giving $\bar{x}_{\ell}=0$ and there are at most $t=|\langle\varepsilon\rangle|$ values of $c \in C^{2^{e}}$ giving $\bar{x}_{\ell}, \bar{x}_{i} \cdot \bar{x}_{j}^{-1} \in\langle\varepsilon\rangle$ for each $\ell=1, \ldots, 2^{e}-1$ and for each pair $(i, j)$ with $1 \leq j<i \leq 2^{e}-1$. On the other hand, it is easy to see that $\bar{x}_{1} \neq 1 \neq \bar{x}_{2}, \bar{x}_{\ell} \neq \varepsilon$ for any $\ell=1, \ldots, 2^{e}-1$ and $\bar{x}_{i} \cdot \bar{x}_{j}^{-1} \neq 1$ for any $1 \leq j<i \leq 2^{e}-1$. Thus, among the values of $C^{2^{e}}$ we have to take off at most

$2^{e}+2(t-2)+\left(2^{e}-3\right)(t-1)+\frac{\left(2^{e}-1\right)\left(2^{e}-2\right)}{2}(t-1)=2^{e-1}\left[\left(2^{e}-1\right) t-\left(2^{e}-3\right)\right]-2$

elements. By the hypothesis on $q,\left|C^{2^{e}}\right|=\frac{q-1}{2^{e}}>2^{e-1}\left[\left(2^{e}-1\right) t-\left(2^{e}-3\right)\right]-2$. Hence the assertion follows from Construction 3.7.

Of course, the bound determined in the previous proposition is as sharp as possible. Nonetheless, in the same way as in Proposition 3.10, we are able to construct an $\mathrm{EA}-\left(K_{81}, C_{20}\right)$-design, even if $81 \ngtr 105$. 
Example 3.11. EA- $\left(K_{81}, C_{20}\right)$-design

In $\mathbb{F}_{81}=\mathbb{Z}_{3}[x] /\left\langle x^{4}+x+2\right\rangle$ we choose $x$ as a primitive element and $\varepsilon=x^{16}=$ $2 x^{3}+x+2$ as a primitive 5-th root of unity in $\mathbb{F}_{81}$. As in Proposition 3.10, we consider the system

$$
\left\{\begin{array}{l}
x_{2}-x_{1}=g\left(x_{1}-1\right) c \\
x_{3}-x_{2}=g^{2}\left(x_{1}-1\right) c \\
\varepsilon-x_{3}=g^{3}\left(x_{1}-1\right) c
\end{array}\right.
$$

Fixing $c=1$ we obtain the solution

$$
\begin{aligned}
& x_{1}=\frac{x^{2}+2 x+2}{x^{3}+x^{2}+x+1}=x^{37}, \quad x_{2}=\frac{2 x^{2}+x+1}{x^{3}+x^{2}+x+1}=x^{77}, \\
& x_{3}=\frac{x^{3}+x^{2}+1}{x^{3}+x^{2}+x+1}=x .
\end{aligned}
$$

It is easy to check that none of the $x_{1} x_{2}^{-1}=x^{40}, x_{1} x_{3}^{-1}=x^{36}, x_{2} x_{3}^{-1}=x^{76}$ belongs to $\langle\varepsilon\rangle$. So, assuming $S=\left\{1, x^{4}\right\}$ as a complete system of representatives for the cosets of $\langle-\varepsilon\rangle$ in $C^{4}$, we can state that

$$
\mathcal{F}=\left\{s\left(1, x_{1}, x_{2}, x_{3}, \varepsilon, x_{1} \varepsilon, x_{2} \varepsilon, x_{3} \varepsilon, \ldots, \varepsilon^{4}, x_{1} \varepsilon^{4}, x_{2} \varepsilon^{4}, x_{3} \varepsilon^{4}\right) \mid s \in S\right\}
$$

is the set of base cycles of an EA- $\left(K_{81}, C_{20}\right)$-design.

\section{Elementary Abelian Cycle Systems of $K_{m \times s}$}

In this section we propose some results about elementary abelian cycle systems of the complete multipartite graph.

As far as the authors are aware, at the moment the only known result about regular cycle systems of $K_{m \times s}$ is the following (see [10] and [26])

Theorem 4.1. A cyclic $k$-cycle system of $K_{m \times k}$ exists if and only if

$$
(m, k) \neq(3,3) \quad \text { and } \quad(m, k) \not \equiv(0,1)(\bmod 2),(2,2)(\bmod 4),(3,2)(\bmod 4) .
$$

Also, it is obvious that removing all lines having a fixed direction from the affine plane of order $q$ one gets a transversal design $T D(q, q)$ (for the well known concept of a transversal design one can see, e.g., [4]) that equivalently can be seen as an EA- $\left(K_{q \times q}, K_{q}\right)$-design. So we can state:

Proposition 4.2. There exists an $E A-\left(K_{p^{n} \times p^{n}}, K_{p^{n}}\right)$-design for every prime $p$.

We start determining a necessary condition for the existence of an EA-cycle system of $K_{m \times s}$. 
Proposition 4.3. If an $E A-\left(K_{m \times s}, C_{k}\right)$-design exists, then

a) $m s=p^{n}$ where $p$ is a prime and $n>1$;

b) $k \leq m s$;

c) $p s(m-1) \equiv 0(\bmod 2 k)$.

Proof. Of course $\left|V\left(K_{m \times s}\right)\right|$ must be a prime power, so let $m s=p^{n}$ where $p$ is a prime and $n>1$. Hence $a$ ) is satisfied. Obviously condition $b$ ) is true. To conclude, we prove $c$ ). For the same reason as in Proposition 3.1, $p^{n-1}$ must be a divisor of the number $\frac{p^{n}\left(p^{n}-s\right)}{2 k}$ of blocks of the required design. Hence $\frac{p s(m-1)}{2 k}$ must be an integer.

\section{Example 4.4. EA- $\left(K_{2 \times 4}, C_{4}\right)$-design}

Let the vertices of $K_{2 \times 4}$ be the elements of $\mathbb{Z}_{2}^{3}$. We choose the following partition of the vertex-set $\{\{000,001,010,011\},\{100,101,110,111\}\}$. Now, it is easy to see that the cycles

$C_{1}=[000,101]_{010}, \quad C_{2}=[101,001]_{010}, \quad C_{3}=[000,110]_{010}, \quad C_{4}=[001,100]_{010}$

form an $\mathrm{EA}-\left(K_{2 \times 4}, C_{4}\right)$-design.

While it is trivial that a $\left(K_{m \times s}, C_{k}\right)$-design together with a $\left(K_{s}, C_{k}\right)$-design gives a $\left(K_{m s}, C_{k}\right)$-design, the following seems not.

Proposition 4.5. If there exist an $E A-\left(K_{m \times s}, C_{k}\right)$-design and an $E A-\left(K_{s}, C_{k}\right)$-design, then there also exists an $E A-\left(K_{m s}, C_{k}\right)$-design.

Proof. Let $\mathcal{A}$ and $\mathcal{B}$ be an EA- $\left(K_{m \times s}, C_{k}\right)$-design and an EA- $\left(K_{s}, C_{k}\right)$-design, respectively, and let $P_{1}, P_{2}, \ldots, P_{m}$ be the parts of $K_{m \times s}$. For any positive integer $n$, let us denote by $I_{n}$ the set $\{1,2, \ldots, n\}$. By hypothesis $m s=p^{n}$ for some odd prime $p$ where $n>1$, so $m=p^{t}$ and $s=p^{r}$ for suitable integers $t$ and $r$. Since $\mathcal{A}$ and $\mathcal{B}$ are elementary abelian, $V\left(K_{m \times s}\right)=\mathbb{Z}_{p}^{t+r}$ and $V\left(K_{s}\right)=\mathbb{Z}_{p}^{r}$. Without loss of generality we can assume that the $m$ parts of $K_{m \times s}$ are the cosets of $\mathbb{Z}_{p}^{r}$ in $\mathbb{Z}_{p}^{t+r}$. Namely, $\left\{g_{1}, g_{2}, \ldots, g_{m}\right\}$ being a complete system of representatives for the cosets of $\mathbb{Z}_{p}^{r}$ in $\mathbb{Z}_{p}^{t+r}, P_{i}=\mathbb{Z}_{p}^{r}+g_{i}$, for any $i \in I_{m}$.

Of course $f_{i}: x \in V\left(K_{S}\right) \rightarrow x+g_{i} \in V\left(K_{P_{i}}\right)$ results in an isomorphism between $K_{s}$ and $K_{P_{i}}$ for any $i \in I_{m}$. Hence, for any $k$-cycle $B \in \mathcal{B}$ we can see that $f_{i}(B)$ results again in a $k$-cycle. Since $\mathcal{F}_{i}=\left\{f_{i}(B) \mid B \in \mathcal{B}\right\}$ is an EA- $k$-cycle system of $K_{P_{i}}$ for any $i \in I_{m}$, it is not hard to see that $\mathcal{A} \cup \mathcal{F}_{1} \cup \mathcal{F}_{2} \cup \ldots \cup \mathcal{F}_{m}$ is an EA- $\left(K_{m s}, C_{k}\right)$-design.

Theorem 4.6. Let $p$ be an odd prime. If there exist an $E A-\left(K_{p^{t} \times p^{r}}, K_{p^{u}}\right)$-design and an $E A-\left(K_{p^{u}}, C_{k}\right)$-design then there also exists an $E A-\left(K_{p^{t} \times p^{r}}, C_{k}\right)$-design.

Proof. Let $\mathcal{A}$ be an EA- $\left(K_{p^{t} \times p^{r}}, K_{p^{u}}\right)$-design and let $\left\{K_{1}, \ldots, K_{\alpha}\right\}$ be a set of representatives for the $\mathbb{Z}_{p}^{t+r}$-orbits on $\mathcal{A}$. As above, by $I_{n}$ we denote the set $\{1,2, \ldots, n\}$. 
By hypothesis there exists an EA- $\left(K_{i}, C_{k}\right)$-design $\mathcal{B}_{i}$, for every $i \in I_{\alpha}$. For any arbitrary block $\Gamma$ of $\mathcal{A}$, there exist $g \in \mathbb{Z}_{p}^{t+r}$ and $i \in I_{\alpha}$ such that $\Gamma=K_{i}+g$, hence $\mathcal{B}_{i}+g=\left\{B+g \mid B \in \mathcal{B}_{i}\right\}$ is an EA- $\left(\Gamma, C_{k}\right)$-design, say $\mathcal{B}_{\Gamma}$. It is not hard to see that $\mathcal{B}=\bigcup_{\Gamma \in \mathcal{A}} \mathcal{B}_{\Gamma}$ is an $\operatorname{EA}-\left(K_{p^{t} \times p^{r}}, C_{k}\right)$-design.

From Theorem 4.6 and Proposition 4.2, applying suitable propositions from Section 3 , we can also state the following

Proposition 4.7. There exists an $E A-\left(K_{p^{n} \times p^{n}}, C_{p t}\right)$-design for every prime $p$ such that $p^{n} \equiv 1(\bmod 2 t)$ with $t=2,3,4$ and $t \leq p^{n-1}$. (cfr. Proposition 3.6).

Proposition 4.8. There exists an $E A-\left(K_{p^{n} \times p^{n}}, C_{p t}\right)$-design for any prime $p$ such that $p^{n} \equiv 1(\bmod 2 t)$ with $\frac{p^{n}-1}{p-1} \geq \frac{t^{2}(t-1)}{2}($ cfr. Proposition 3.3$)$.

Proposition 4.9. Let $p^{n}=p^{d r}$ be an odd prime power, where $r>1$. There exists an $E A-\left(K_{p^{n} \times p^{n}}, C_{p t}\right)$-design for every odd integer $t$ dividing $p^{d}-1$ (cfr. Proposition 3.5).

Proposition 4.10. There exists an $E A-\left(K_{p^{n} \times p^{n}}, C_{k}\right)$-design for any prime $p$ such that $p^{n} \equiv 1(\bmod 2 k)$ and any $k \equiv 2(\bmod 4)$, with $k \neq 2(\mathrm{cfr}$. Proposition 3.8$)$.

Proposition 4.11. Let $q \equiv 1(\bmod 2 k)$ be a prime power, where $k=2^{e} t$ with $e>1$ and $t$ odd. There exists an $E A-\left(K_{q \times q}, C_{k}\right)$-design for any $q>2^{2 e-1}\left[\left(2^{e}-1\right) t-\left(2^{e}-\right.\right.$ $3)]-2^{e+1}+1$ (cfr. Proposition 3.10).

Acknowledgements. The authors are grateful to Prof. Marco Buratti for helpful suggestions on the item of this paper and to the referee for providing valuable remarks.

\section{References}

1. Adams, P., Billington, E.J.: Completing some spectra for 2-perfect cycle systems. Austral. J. Combin. 7, 175-187 (1993)

2. Alspach, B., Gavlas, H.: Cycle decompositions of $K_{n}$ and $K_{n}-I$. J. Combin. Theory Ser. B 81, 77-99 (2001)

3. Bennett, F.E., Ge, G., Zhu, L.: Existence of Steiner seven-cycle systems. Discrete Math. 252, 1-16 (2002)

4. Beth, T., Jungnickel, D., Lenz, H.: Design Theory. Cambridge University Press, (1999)

5. Bryant, D., El-Zanati, S.: Graph decompositions. In: Handbook of Combinatorial Designs, Second Edition. Colbourn, C.J., Dinitz, J.H., (eds.), Chapman \& Hall/CRC, Boca Raton, FL, pp. 477-486 (2006)

6. Bryant, D., Rodger, C.A.: Cycle decompositions. In: Handbook of Combinatorial Designs, Second Edition. Colbourn, C.J., Dinitz, J.H., (eds.), Chapman \& Hall/CRC, Boca Raton, FL, pp. 373-382 (2006)

7. Bonisoli, A., Buratti, M., Mazzuoccolo, G.: Doubly transitive 2-factorizations. J. Combin. Designs 15, 120-132 (2007)

8. Buratti, M.: Rotational $k$-cycle systems of order $v<3 h$; another proof of the existence of odd cycle systems. J. Combin. Designs 11, 433-441 (2003) 
9. Buratti, M.: Cycle decompositions with a sharply vertex transitive automorphism group. Le Matematiche (Catania) 59, 91-105 (2004)

10. Buratti, M., Del Fra, A.: Existence of cyclic $k$-cycle systems of the complete graph. Discrete Math. 261, 113-125 (2003)

11. Buratti, M., Del Fra, A.: Cyclic hamiltonian cycle systems of the complete graph. Discrete Math. 279, 107-119 (2004)

12. Buratti, M., Rania, F., Zuanni, F.: Some constructions for cyclic perfect cycle systems. Discrete Math. 299, 33-48 (2005)

13. Granville, A., Moisiadis, A., Rees, R.: Nested Steiner $n$-cycle systems and perpendicular array. J. Combin. Math. Combin. Comput. 3, 163-167 (1988)

14. Kotzig, A.: Decomposition of a complete graph into $4 k$-gons. Mat. Fyz. Casopis Sloven. Akad. Vied 15, 229-233 (1965) (in Russian)

15. Lindner, C.C., Rodger, C.A.: 2-perfect $m$-cycle systems. Discrete Math. 104, 83-90 (1992)

16. Lindner, C.C., Stinson, D.R.: Steiner pentagon systems. Discrete Math. 52, 67-74 (1984)

17. Manduchi, E.: Steiner heptagon systems. Ars Combin. 31, 76-85 (1991)

18. Peltesohn, R.: Eine losung der beiden heffterschen differenzenprobleme. Compos. Math. 6, 251-257 (1938)

19. Rosa, A.: On cyclic decompositions of the complete graph into $(4 m+2)$-gons. Mat. Fyz. Casopis Sloven. Akad. Vied 16, 349-352 (1966)

20. Rosa, A.: On cyclic decompositions of the complete graph into polygons with odd number of edges (Slovak). Casopis Pest. Mat. 91, 53-63 (1966)

21. Rosa, A.: On decompositions of a complete graph into $4 n$-gons. Mat. Casopis Sloven. Akad. Vied 17, 242-246 (1967) (in Russian)

22. S̃ajna, M.: Cycle decompositions of $K_{n}$ and $K_{n}-I$. Ph.D. Thesis, Simon Fraser University, July (1999)

23. Sajna, M.: Cycle decompositions III: complete graphs and fixed length cycles. J. Combin. Designs 10, 27-78 (2002)

24. Vietri, A.: Cyclic $k$-cycle systems of order $2 k n+k$; a solution of the last open cases. J. Combin Designs 12, 299-310 (2004)

25. Vietri, A.: Difference families in $\mathbb{Z}_{2 d+1} \oplus \mathbb{Z}_{2 d+1}$ and infinite translation designs in $\mathbb{Z} \oplus \mathbb{Z}$. Graphs and Comb. 23, 111-121 (2007)

26. Wu, S.L., Fu, H.L.: A note on cyclic $m$-cycle systems of $K_{r(m)}$. Graphs and Combin. 22, 427-432 (2006)

Received: February 12, 2008

Final version received: October 25, 2008 